\title{
Fox H-function: a Study Case on Variate Modeling of Dual-Hop Relay Over Weibull Fading Channels
}

\author{
Carlos H. M. de Lima*, Hirley Alves ${ }^{\dagger}$ and Pedro. H. J. Nardelli ${ }^{\ddagger}$ \\ * São Paulo State University (UNESP), São João da Boa Vista, Brazil \\ ${ }^{\dagger}$ Centre for Wireless Communications (CWC), University of Oulu, Finland \\ ‡Laboratory of Control Engineering and Digital Systems, Lappeenranta University of Technology, Finland \\ carlos.lima@sjbv.unesp.br, hirley.alves@oulu.fi,pedro.nardelli@lut.fi
}

\begin{abstract}
This paper proposes a novel analytical framework based on the Fox $\mathrm{H}$-function distribution. To test its efficacy we focus on a study case where we derive closed form expressions for the distribution of the end-to-end signal to noise ratio, and corresponding outage probability for the dual-hop amplify-andforward configuration over Weibull-fading channels with channel state information. An extensive simulation campaign was carried out to corroborate the proposed approach.
\end{abstract}

Index Terms-Machine-to-machine, $\mathrm{H}$-function distribution, amplify-and-forward, outage probability

\section{INTRODUCTION}

To fully enable the coexistence of human and machine type communications in $5 \mathrm{G}$ and beyond systems, not only the already established procedures need to be revised, but also several new technologies have to be introduced. More specifically, allowing a large number of machines and legacy human type communication to simultaneously transmit and share the air interface requires further development of cooperative mechanisms. This new context requires new analytical models so as to capture the various non-identical propagation characteristics of radio channel [1], [2].

Cooperative schemes have already been proposed for device-to-device communication, proving that it can reduces energy consumption of low-power machine type terminals while enhance reliability and throughput. In similar way, Machine-to-Machine (M2M) relaying can also reduce energy expenditure for cell-edge machine type terminals [3]. In both cases, the radio link between source, relay and destination may exhibit distinct characteristics.

In [4], the authors analyzed the performance of digital modulations on Weibull fading channels and derived new closed-form expressions for the respective moment generating function with integer value fading parameters. Similarly, Hasna et al. studied the End-to-End (E2E) performance of dual-hop relaying over Rayleigh-fading channels [5], where closed form expressions for the statistic of the harmonic mean of two independent exponential variates were derived, as well as comparisons between regenerativeand non-regenerative systems were derived. Along the same line, the authors in [6] evaluated the outage probability of dual-hop relaying over Nakagami- $m$ fading channels; the exact analytical expression of the outage probability for non integer $m$ parameters were obtained by means of bivariate $\mathrm{H}-$ and $\mathrm{G}$-functions.
Motivated by these results, we introduce here a new framework to derive closed form expressions for the Probability Density Function (PDF) and Cumulative Distribution Function (CDF) (outage probability) of the E2E Signal to Noise Ratio (SNR) distribution for the dual hop Channel State Information (CSI) assisted Amplify and Forward (AF) relaying over Weibull fading channels. As discussed in [4], the Weibull distribution offers a convenient model to capture the effects of multi path fading channels in indoor [7] and outdoor [8] radio propagation environments. Measurements in vehicle-tovehicle urban channels also suggest a multipath fading with Weibull distribution [9]. To derive our analytical expressions, we resort to Fox $\mathrm{H}$-function distribution (a generalization of hypergeometric functions given in term of contour integrals involving products of gamma functions) and the well known moment matching approach [11], [12]. The idea of using Fox $\mathrm{H}$-functions to establish a unified framework to model radio channel statistics [13], [14] and evaluate the performance of wireless communication systems [15] is not totally new. However, the $\mathrm{H}$-function distribution was only introduced in [12] along with its properties and special cases. Recently, [16] analyzes the error probability and capacity at high and low SNR regimes using algebraic asymptotic expansions of the $\mathrm{H}-$ transform. Herein, we use $\mathrm{H}$-function distribution to represent the Weibull multi-path fading and then compute closed form expression for the corresponding outage probability.

Our contributions are summarized as follows:

- We introduced a novel analytical framework based on the Fox $\mathrm{H}$-function distribution [11] for computing the distribution of the E2E SNR in cooperative AF scenarios.

- We derive the closed-form expressions for the outage probability for independent, but not necessarily identically distributed Weibull variates.

- We propose a closed-form approximation for the sum of independent, but not necessarily identically distributed Weibull variates.

The reminder of this paper is organized as follows. Section II introduces the mathematical concepts used to derived our analytical framework based on the Fox $\mathrm{H}$-function, Mellin transform and its main properties. Thereafter, our framework is applied to the dual hop relay problem in III. We then use Fox $\mathrm{H}$-function variates to derive closed form expressions for 
the dual-hop relaying E2E SNR distribution. The outage probability is shown in for various radio channel configurations between source, relay and destination channels. In Section VI, final observations and conclusions are provided.

\section{PRELIMINARIES AND ANALYTICAL FrAMEWORK}

We introduce here the proposed analytical framework for evaluating the performance of wireless networks under various fading channel regimes [17]. We first discuss Mellin transforms that are used to conveniently carry out the algebra of random variables [18]. Then, the Fox $\mathrm{H}$-function distribution is defined based on a Mellin-Barnes integral [19]. Various identities and properties that are very useful in manipulating $\mathrm{H}$-functions are introduced as well.

\section{A. Mellin Transform and General Formulas}

Definition 1: (Mellin transform) The Mellin Transform (MT) [20], [21] of a Random Variable (RV) $Z$ whose PDF is given by $f_{Z}(z)$ is defined on the $(0, \infty)$ as,

$$
\mathscr{M}_{s}\left\{f_{Z}(z)\right\} \triangleq \int_{0}^{\infty} f_{Z}(z) z^{s-1} \mathrm{~d} z \triangleq \mathrm{E}\left[Z^{s-1}\right]
$$

where $s \in \mathbb{C}$ and $\mathrm{E}[\cdot]$ denotes expectation.

Property 1: Let $g(x)$ be a complex-valued function that exists over $(0, \infty)$ and is locally integrable. The MT of $g(a x)$ with $a \in \mathbb{C}$ is derived as

$$
\begin{aligned}
\mathscr{M}_{s}\{g(a x)\} & =\int_{0}^{\infty} g(a x) x^{s-1} \mathrm{~d} x \\
& \stackrel{(i)}{=} a^{-s} \int_{0}^{\infty} g(y)(y)^{s-1} \mathrm{~d} y \\
& =a^{-s} \mathscr{M}_{s}\{g(x)\},
\end{aligned}
$$

where step $(i)$ comes from the change of variable $y=a x$.

Property 2: The MT of $x^{p} g(x)$ is derived as

$$
\begin{aligned}
\mathscr{M}_{s}\left\{x^{p} g(x)\right\} & =\int_{0}^{\infty} x^{p} g(x) x^{s-1} \mathrm{~d} x \\
& =\int_{0}^{\infty} g(x) x^{s+p-1} \mathrm{~d} x \\
& =\mathscr{M}_{s+p}\{g(x)\} .
\end{aligned}
$$

\section{B. The Fox H-Function}

Definition 2: (the Fox $\mathrm{H}$-function) The Fox $\mathrm{H}$-function is defined by means of a Mellin-Barnes type integral in the following form [11], [12], [18]:

$$
\begin{aligned}
& \mathrm{H}(z)=\mathrm{H}_{p, q}^{m, n}\left[z \mid \begin{array}{l}
\left(a_{i}, A_{i}\right)_{1, p} \\
\left(b_{j}, B_{j}\right)_{1, q}
\end{array}\right] \\
& =\frac{1}{2 \pi \imath} \int_{\mathfrak{L}} \frac{\prod_{j=1}^{m} \Gamma\left[b_{j}+B_{j} s\right] \prod_{i=1}^{n} \Gamma\left[1-a_{i}-A_{i} s\right] z^{-s} \mathrm{~d} s}{\prod_{i=n+1}^{p} \Gamma\left[a_{i}+A_{i} s\right] \prod_{j=m+1}^{q} \Gamma\left[1-b_{j}-B_{j} s\right]},
\end{aligned}
$$

where $\Gamma[\cdot]$ is the gamma function $[22, \S 6], z, c, a_{i}, b_{j} \in \mathbb{C}$, $A_{i}, B_{j} \in \mathbb{R}^{+}$, and $m, n, p, q \in \mathbb{Z}$ such that $1 \leqslant j \leqslant m, 1 \leqslant$ $i \leqslant n, 0 \leqslant m \leqslant q, 0 \leqslant n \leqslant p, A_{i}>0, B_{j}>0 ; \mathfrak{L}$ is a contour in the complex $s$-plane ranging from $\omega-\imath \propto 0$ to $\omega+\imath \infty 0$ so that all left half-plane poles of $\prod_{j=1}^{m} \Gamma\left[b_{j}+B_{j} s\right]$ lie to the left of $\mathfrak{L}$ and all right half-plane poles of $\prod_{i=1}^{n} \Gamma\left[1-a_{i}-A_{i} s\right]$ lie to the right.

The following properties, whose derivations are detailed in [11], are important to operate with the $\mathrm{H}$-function.

Property 3: For $\sigma \in \mathbb{C}$, there holds the relation,

$$
z^{\sigma} \mathrm{H}_{p, q}^{m, n}\left[z \mid \begin{array}{l}
\left(a_{i}, A_{i}\right)_{1, p} \\
\left(b_{j}, B_{j}\right)_{1, q}
\end{array}\right]=\mathrm{H}_{p, q}^{m, n}\left[z \mid \begin{array}{c}
\left(a_{i}+\sigma A_{i}, A_{i}\right)_{1, p} \\
\left(b_{j}+\sigma B_{j}, B_{j}\right)_{1, q}
\end{array}\right] .
$$

Property 4: For $k>0$, we also have the following,

$$
\mathrm{H}_{p, q}^{m, n}\left[z \mid \begin{array}{l}
\left(a_{i}, A_{i}\right)_{1, p} \\
\left(b_{j}, B_{j}\right)_{1, q}
\end{array}\right]=k \mathrm{H}_{p, q}^{m, n}\left[z^{k} \mid \begin{array}{l}
\left(a_{i}, k A_{i}\right)_{1, p} \\
\left(b_{j}, k B_{j}\right)_{1, q}
\end{array}\right] .
$$

\section{H-Function Distribution and its Raw Moments}

Definition 3: (the Fox H-function distribution) The PDF of an arbitrary $\mathrm{RV} Z$ is given by,

$$
f_{Z}(z)=\left\{\begin{array}{l}
k \mathrm{H}_{p, q}^{m, n}\left[c z \mid \begin{array}{l}
\left(a_{i}, A_{i}\right)_{1, p} \\
\left(b_{j}, B_{j}\right)_{1, q}
\end{array}\right], z>0 \\
0, \text { otherwise }
\end{array}\right.
$$

where the constant $k$ normalizes the area under the density function (over the appropriate range) to unity [23].

The Fox H-Function Distribution in (4) is interesting because it covers many non-negative distributions, such as Gamma, Beta, Exponential, Rayleigh and Weibull [12]. In other words, they can be readily expressed in the form of (7). Moreover, many characteristics of probability distributions such as moments, CDF, integral transforms (Laplace, Fourier, and Mellin) are easily obtained from such representation.

Property 5: Considering that a real valued RV $Z$ can be represented as a $\mathrm{H}$-function variate in (7), the $r$ th (non-central) moment of $Z$ is easily derived using the Mellin transform as

$$
\begin{aligned}
& \mathrm{E}\left[Z^{r}\right]=\mathscr{M}_{r+1}\left\{f_{Z}(z)\right\} \\
& =\frac{k}{c^{r+1}} \frac{\prod_{j=1}^{m} \Gamma\left[b_{j}+B_{j}+B_{j} r\right] \prod_{i=1}^{n} \Gamma\left[1-a_{j}-A_{j}-A_{j} r\right]}{\prod_{i=n+1}^{p} \Gamma\left[a_{j}+A_{j}+A_{j} r\right] \prod_{j=m+1}^{q} \Gamma\left[1-b_{j}-B_{j}-B_{j} r\right]} .
\end{aligned}
$$

Proof: The $r$ th moment about the origin of $f_{Z}(z)$ is given by, $\mathrm{E}\left[Z^{r}\right]=\int_{0}^{\infty} z^{r} f_{Z}(z) \mathrm{d} z$. Provided that the integration converges and the $f_{Z}(z)$ can be expressed in terms of the Fox $\mathrm{H}$-function, the $r$ th moment using the MT is given by, $\mathrm{E}\left[Z^{r}\right]=\mathscr{M}_{r}\left\{z^{r} f_{Z}(z)\right\}$. Finally, using Properties 1 and 2, we obtain (8).

\section{System MODEL}

To assess the end-to-end performance of the source-relay configuration we assume that nodes operate in half-duplex mode with omni-directional antenna radiation pattern, in which the relay lies in a straight line between source and destination. We assume a discrete-time channel with stationary and ergodic time-varying gain denoted by $x[t]$. Signal transmissions occur 
over slow, frequency non-selective Weibull fading channels. The received signal is given by

$$
y_{i}[t]=\alpha_{i} x[t]+n_{i}[t],
$$

where $\alpha_{i}$ yields the fading channel amplitude between communicating peers, $x[t]$ is the signal transmitted by the source with unity power $\mathrm{E}\left[x^{2}\right]=1$, and $n_{i}[t]$ is an AWGN noise signal with one-sided power spectral density $N_{0}$.

The channel gain (signal squared envelope) follows a Weibull distribution with parameters $(\lambda, \beta)$ with PDF

$$
f_{Z}(z)=\lambda \beta z^{\beta-1} \exp \left(-\lambda z^{\beta}\right),
$$

where the parameter $\lambda$ is related to the average fading power $\mathrm{E}\left[\alpha_{i}\right]$ given by $\lambda_{i}=\left(\frac{\mathrm{E}\left[\alpha_{i}^{2}\right]}{\Gamma\left[1+2 / \theta_{i}\right]}\right)^{\theta_{i} / 2}$.

The $k$-th power of a Weibull distributed variate with parameters $(\lambda, \beta)$ is also a Weibull-distributed with $(\lambda / k, \beta)$ [4]. Let $E_{S}$ be the average symbol energy, then, the average SNR per symbol for the $i$ th hop is given by $\gamma_{i}=\frac{E_{S}}{N_{0}} \alpha_{i}^{2}$. Note that both the average noise power and the average symbol energy are considered to be the unity.

\section{A. Weibull as a special case of the $\mathrm{H}-$ function distribution}

The Weibull distribution PDF (10) can be written by means of the $\mathrm{H}$-function representation (3) as

$$
f_{Z}(z)=\left\{\begin{array}{c}
\lambda^{1 / \beta} \mathrm{H}_{0,1}^{1,0}\left[\left.\lambda^{1 / \beta} z\right|_{(1-1 / \beta, 1 / \beta)}\right], z>0, \\
0, \text { otherwise }
\end{array}\right.
$$

where $\beta>0$ is the shape parameter and $\sqrt{1 / \lambda}>0$ is the scale parameter.

To plot the PDF of the Weibull distribution from the $\mathrm{H}$-function representation, we need to compute the contour integral in (3) through a doubly exponential quadrature [24], [25]. Fig. 1 illustrates the PDF of a Weibull distribution using the $\mathrm{H}$-function representation. For this plot, we consider the shape $(\beta)$ and scale parameters $(1 / \lambda)$ equal to 6 and $1 / 2$.

In the next section, we extend this framework to deal with more elaborate algebra between RVs taking advantage of this representation.

\section{Application to the Dual hop Relay Scenario}

\section{A. End-to-End SNR Distribution}

We consider a scenario where nodes communicate through CSI-assisted AF relaying over Weibull fading channels [6]. The end-to-end SNR expression in this case is given by [5]:

$$
\gamma_{\mathrm{end}}=\frac{\gamma_{1} \gamma_{2}}{\gamma_{1}+\gamma_{2}} \stackrel{(i)}{=}\left(\frac{1}{\gamma_{1}}+\frac{1}{\gamma_{2}}\right)^{-1}
$$

where $\gamma_{i}$ is the SNR of the $i$ th radio link (hop).

To derive (12) using the $\mathrm{H}$-function distribution, we make use of the mathematical framework introduced in Section II which allow us to compute the harmonic mean (as in step (i)) of two independent and non-identically distributed (i.n.i.d) Weibull variates. Hereafter, we present the formulation needed to obtain the end to end SNR in (12).

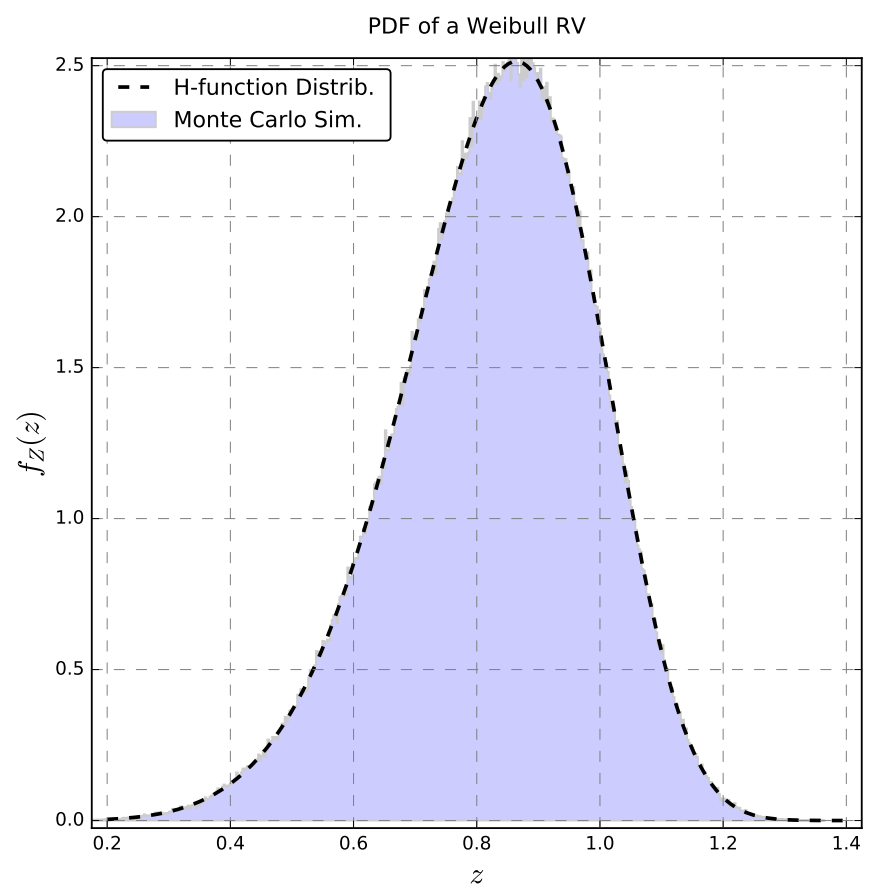

Fig. 1. PDF of a Weibull distribution with parameters $\lambda$ and $\beta$ using the $\mathrm{H}$-function representation.

\section{B. Reciprocal of Fox $\mathrm{H}-$ Function Variates}

To obtain the PDF of the reciprocal of a Weibull distribution in (11), we derive the distribution of a rational power of the $\mathrm{H}$-function variate. Then, we use [12, Theorem 4.2] and Property 4 to derive the reciprocal as $Y=Z^{\eta}$, for $\eta<0$, thus

$$
f_{Y}(y ; \lambda, \beta)=\lambda^{-1 / \beta} \mathrm{H}_{1,0}^{0,1}\left[\left.\lambda^{-1 / \beta} y\right|_{(-1 / \beta, 1 / \beta)} ^{-}\right] .
$$

Fig. 2 shows the reciprocal of the Weibull distribution using the $\mathrm{H}$-function representation, in which we consider the same configuration parameters previously used in Section III-A.

\section{Distribution of the Sum of Two i.n.i.d $\mathrm{H}$-function Variates}

To calculate (12), we need to compute the sum $W=Y_{1}+$ $Y_{2}$. Therefore, moment-match approach is used to derive the sum of two independent $\mathrm{H}$-function variates [26].

Proposition 1: Consider two mutually independent $\mathrm{H}-$ function variates $Y_{1}$ and $Y_{2}$. The $r$ th moment about the origin is computed using the binomial formula

$$
\mu_{r} \sum_{i=0}^{r}\left(\begin{array}{l}
r \\
i
\end{array}\right) \mathrm{E}\left[Y_{1}^{r-i} \quad\right] \mathrm{E}\left[Y_{2}^{i}\right]
$$

Proof: Let $\mu_{r}=\mathrm{E}\left[W^{r}\right]=\mathrm{E}\left[\left(Y_{1}+Y_{2}\right)^{r}\right]$, then using (8), we build a nonlinear system of $2(p+q)+2$ equations so as to identify the parameters the approximating $\mathrm{H}$-function distribution. Note that [26] shows that it is possible to eliminate the parameters $k$ and $c$ from the system of equations through algebraic manipulation. To solve the system of nonlinear equations, we use the modified Powell method [27]. 


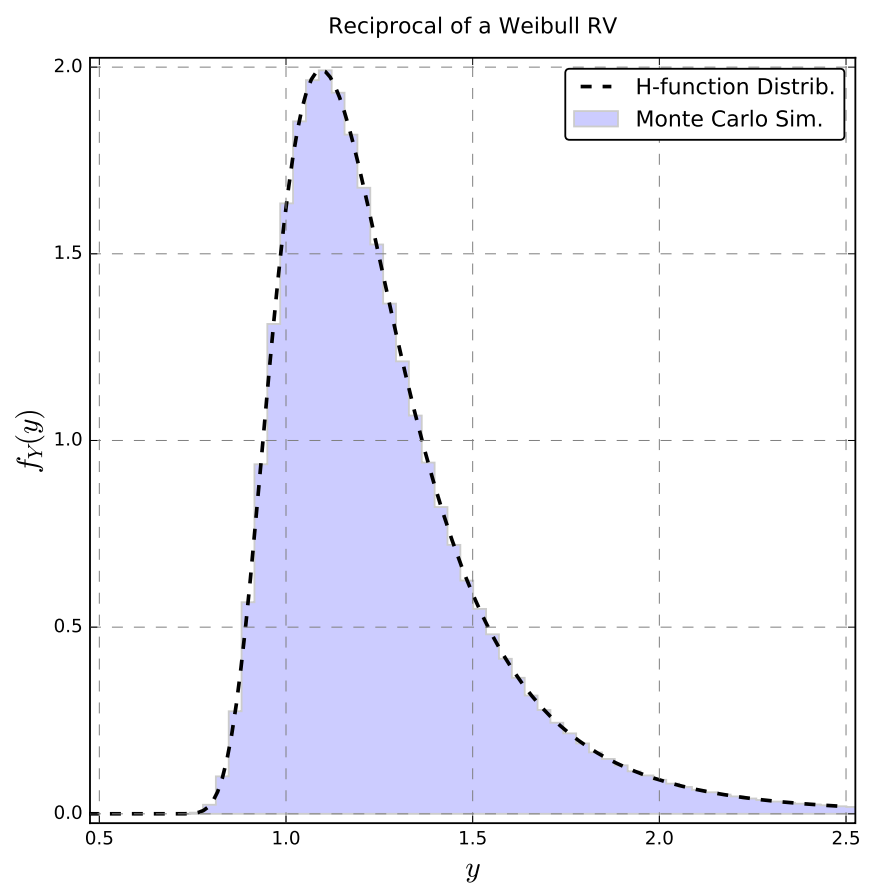

Fig. 2. PDF of a Weibull distribution with parameters $\lambda$ and $\beta$ using the $\mathrm{H}-$ function representation.

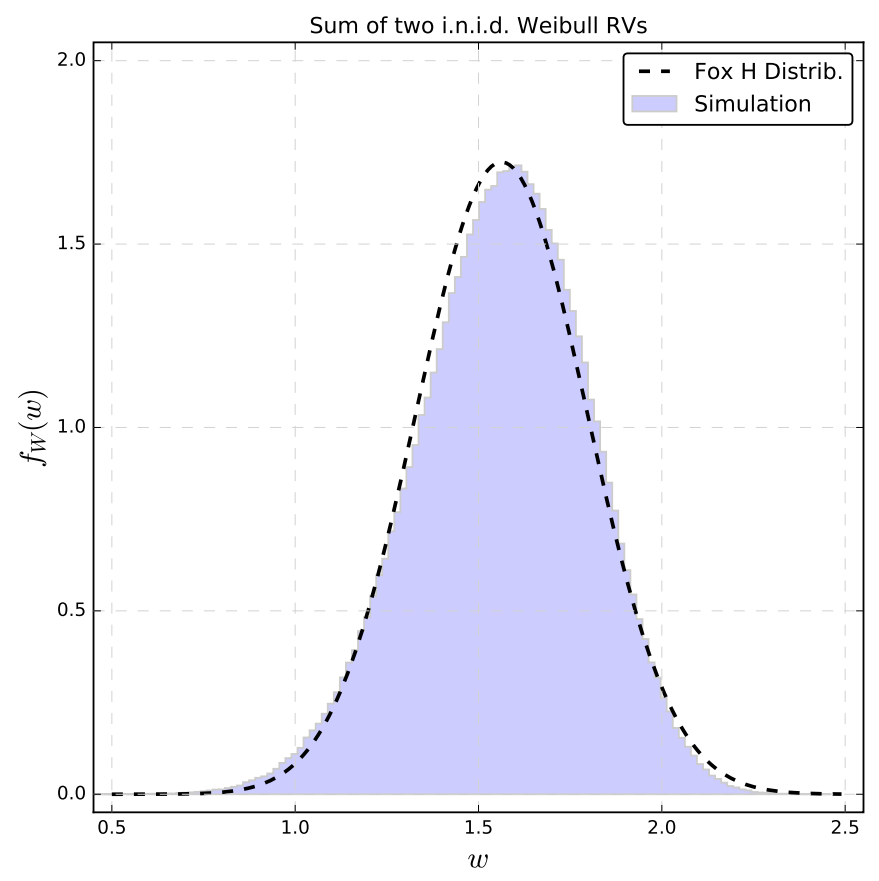

Fig. 3. PDF of the sum of two independent not identically distributed Weibull RVs.

Fig. 3 shows the PDF of the sum of two independent not identically distributed Weibull RVs. considering the following pairs of configuration parameters, $\left(\beta_{1}, \lambda_{1}\right)=(6,2)$ and $\left(\beta_{2}, \lambda_{2}\right)=(5,3)$.

\section{PDF of the E2E SNR}

Using the binomial formula in (14), we can obtain the sum of i.n.i.d RVs $W=Y_{1}+Y_{2}$. Thus, to obtain the end-to-end SNR performance, we need to compute $Z=W^{-1}$ using the formulation in (13). Since both variates in this sum are of type [0 1110$]$ (refer to the sub- and super-scripts of $H$ ), we fit the sum to another $\mathrm{H}$-function distribution with the same parameters [0 1110$]$, as follows

$$
f_{Z}(z ; \lambda, \beta)=k_{z} \mathrm{H}_{1,0}^{0,1}\left[c_{z} z \mid \begin{array}{c}
\left(a_{1}, A_{1}\right) \\
-
\end{array}\right] .
$$

From Property 5 the corresponding moments are:

$$
\mu_{r}(z)=\frac{k_{z}}{c_{z}^{r+1}} \Gamma\left[1-a_{1}-A_{1}-A_{1} r\right] .
$$

Then, we consider the first four moments and use a modified version of the Powell hybrid method to solve the resulting system of equations [28].

Finally, to determine the SNR we just need to derive the reciprocal $W=Z^{-1}$ using (13) as follows

$$
f_{W}(w ; \lambda, \beta)=\frac{k_{z}}{c_{z}^{2}} \mathbf{H}_{0,1}^{1,0}\left[\frac{w}{c_{z}} \mid \begin{array}{c}
- \\
\left(1-a_{1}-2 A_{1}, A_{1}\right)
\end{array}\right] .
$$

\section{E. CDF of the E2E SNR}

The CDF of a $\mathrm{H}$-function distribution is given as $F_{Z}(z)=$ $\int_{0}^{z} \mathrm{H}(u) \mathrm{d} u$. Using the Laplace transform of a $\mathrm{H}$-function distribution and its inverse [29], we find that $F_{Z}(z)$ can also be presented in the $\mathrm{H}$-function following format [23]:

$$
\begin{gathered}
F_{Z}(z ; \lambda, \theta)=\frac{k_{W}}{c_{W}} \mathrm{H}_{2,2}^{1,1}\left[c_{W} z \mid \begin{array}{c}
(1,1) \\
\left(1-a_{1}-A_{1}, A_{1}\right),(0,1)
\end{array}\right] . \\
\text { V. NUMERICAL RESULTS }
\end{gathered}
$$

We evaluate here the end-to-end SNR performance of the cooperative system presented in Section III. To corroborate the proposed analytical framework, we use Monte Carlo simulations $\left(10^{6}\right.$ snapshots). We consider i.n.i.d $\mathrm{H}$-function variates and variable power ratio between the two-hop links.

Fig. 4 shows the CDF of the distribution of the E2E SNR when the communication links source, relay and destination are not identically distributed, and with CSI at the relay node. The $\mathrm{H}$-function representation provides a very good match to the simulation results obtained throughout Monte Carlo simulation for the entire range of evaluated SNR values.

\section{Conclusions And Final Remarks}

In this paper, we introduced an analytical framework to compute the performance of communication systems that are subject to different radio channel characteristics. The proposed approach uses the Fox $\mathrm{H}$-function distribution that incorporates many traditional fading models like Weibull and Nakagami. To illustrate its strength, we assessed the performance of the AF dual-hop relay scenario with perfect CSI. We assume fading channels modeled as independent but not necessarily identical Weibull variates, deriving a closed form expression for the outage probability. 


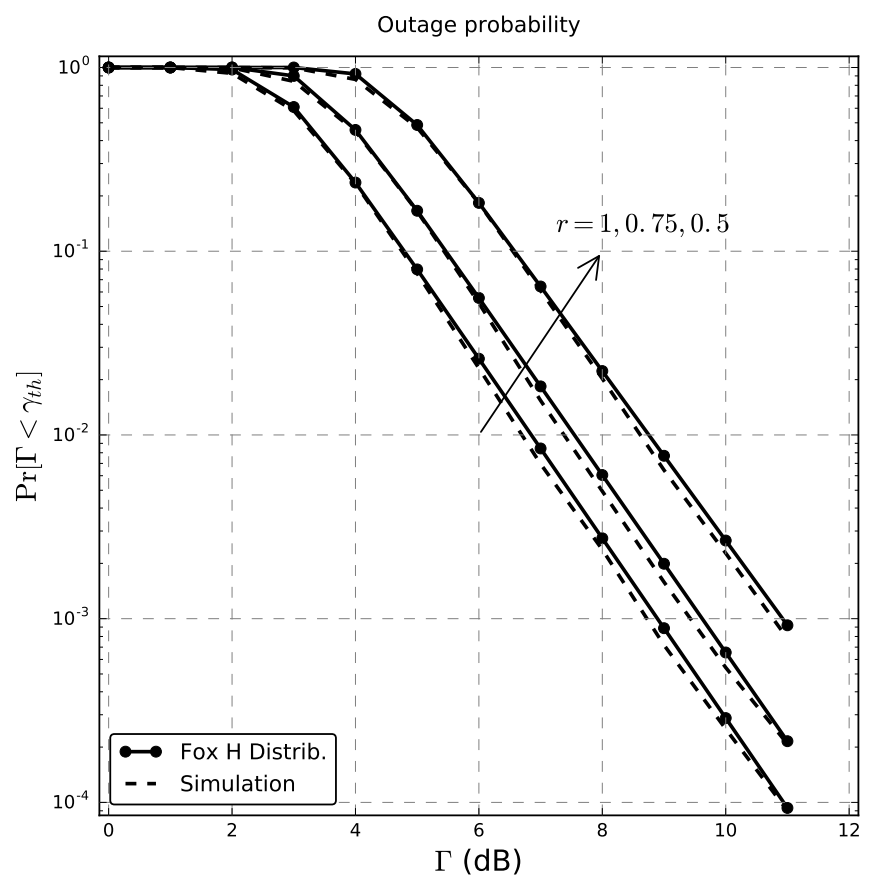

Fig. 4. E2E Outage probability for the dual hop amplify and forward channel in (12), whose CDF is given in closed-from in (18), for different rate $r$ requirements.

More importantly, the proposed framework may be used to derive closed form expressions for different scenarios in a relatively simple manner. It is worth saying the $\mathrm{H}$-function representation simplifies the analysis, while complicates the numerical solution since it involves nowadays a kind of "handcraft" procedure. Although this may impose some technical difficult, the $\mathrm{H}$-function distribution allows for closed form analytical solutions and the problem moves to how to compute them. In future works, we plan to employ the proposed approach to different scenarios including different cooperative strategies, multiple hop communication, random positions and different fading channels.

\section{ACKNOWLEDGMENT}

The research leading to these results has received funding from Strategic Research Council/Aka BCDC Energy (Grant n.292854), Aka/SAFE (Grant n.303532) and CNPq (Universal n. $432925 / 2016-3)$.

\section{REFERENCES}

[1] H. Shariatmadari et al., "Machine-type communications: current status and future perspectives toward 5G systems," IEEE Communications Magazine, vol. 53, no. 9, pp. 10-17, September 2015.

[2] N. Nomikos, D. N. Skoutas, and P. Makris, "Relay selection in $5 \mathrm{~g}$ networks," in International Wireless Communications and Mobile Computing Conference, Aug 2014, pp. 821-826.

[3] S. Andreev et al., "Understanding the IoT connectivity landscape: a contemporary $\mathrm{M} 2 \mathrm{M}$ radio technology roadmap," IEEE Communications Magazine, vol. 53, no. 9, pp. 32-40, September 2015.

[4] J. Cheng, C. Tellambura, and N. Beaulieu, "Performance analysis of digital modulations on Weibull fading channels," in Vehicular Technology Conference, 2003. VTC 2003-Fall. 2003 IEEE 58th, vol. 1, Oct. 2003, pp. 236-240 Vol.1.
[5] M. Hasna and M.-S. Alouini, "End-to-end performance of transmission systems with relays over Rayleigh-fading channels," Wireless Communications, IEEE Transactions on, vol. 2, no. 6, pp. 1126-1131, Nov 2003.

[6] M. Xia, Y. C. Wu, and S. Aissa, "Exact outage probability of dualhop csi-assisted af relaying over nakagami- $\mathrm{m}$ fading channels," IEEE Transactions on Signal Processing, vol. 60, no. 10, pp. 5578-5583, Oct 2012.

[7] H. Hashemi, "The indoor radio propagation channel," Proceedings of the IEEE, vol. 81, no. 7, pp. 943-968, Jul 1993.

[8] G. Tzeremes and C. G. Christodoulou, "Use of Weibull distribution for describing outdoor multipath fading," in IEEE Antennas and Propagation Society International Symposium (IEEE Cat. No.02CH37313), vol. 1, 2002, pp. 232-235 vol.1.

[9] Y. Ibdah and Y. Ding, "Mobile-to-mobile channel measurements at 1.85 $\mathrm{GHz}$ in suburban environments," IEEE Trans. Commun., vol. 63, no. 2, pp. 466-475, Feb 2015.

[10] M. You, H. Sun, J. Jiang, and J. Zhang, "Effective rate analysis in weibull fading channels," IEEE Wireless Commun. Lett., vol. 5, no. 4, pp. 340-343, Aug 2016.

[11] A. A. Kilbas, H-Transforms: Theory and Applications. CRC Press, 2004.

[12] B. D. Carter and M. D. Springer, "The distribution of products, quotients and powers of independent H-Function variates," SIAM Journal on Applied Mathematics, vol. 33, no. 4, pp. 542-558, Dec. 1977.

[13] B. A. Dolan, "The mellin transform for moment-generation and for the probability density of products and quotients of random variables," Proceedings of the IEEE, vol. 52, no. 12, pp. 1745-1746, Dec 1964.

[14] K. Yao, M. K. Simon, and E. Bigiieri, "Unified theory on wireless communication fading statistics based on SIRP," in IEEE 5th Workshop on Signal Processing Advances in Wireless Communications, 2004, pp. 135-139.

[15] F. Yilmaz and M.-S. Alouini, "A unified MGF-based capacity analysis of diversity combiners over generalized fading channels," IEEE Trans. Commun., vol. 60, no. 3, pp. 862-875, 2012.

[16] Y. Jeong, H. Shin, and M. Z. Win, "H-transforms for wireless communication," IEEE Trans. Inf. Theory, vol. 61, no. 7, pp. 3773-3809, July 2015.

[17] L. J. Slater, Generalized hypergeometric functions. Cambridge, 1966.

[18] M. D. Springer, The Algebra of Random Variable. Wiley \& Sons Inc, 1979.

[19] C. Fox, "The G and H functions as symmetrical Fourier kernels," Transactions of the American Mathematical Society, vol. 98, no. 3, pp. 395-429, 1961.

[20] I. S. Gradshteyn and I. M. Ryzhik, Table of Integrals, Series, and Products, A. Jeffrey and D. Zwillinger, Eds. Academic Press, 2007.

[21] A. D. Poularikas, Transforms and applications handbook. CRC Press, 2010.

[22] M. Abramowitz and I. A. Stegun, Handbook of Mathematical Functions with Formulas, Graphs, and Mathematical Tables, 9th ed. Dover, 1965.

[23] C. D. Bodenschatz, R. A. Boedigheimer, and I. D. Cook Jr, "New and generalized h-function distributions," American Journal of Mathematical and Management Sciences, vol. 10, no. 1-2, pp. 185-196, 1990.

[24] A. Meurer et al., "Sympy: symbolic computing in python," PeerJ Computer Science, vol. 3, p. e103, Jan. 2017. [Online]. Available: https://doi.org/10.7717/peerj-cs.103

[25] F. Johansson et al., mpmath: a Python library for arbitraryprecision floating-point arithmetic (version 0.18), December 2013, http: / /mpmath.org/.

[26] C. D. Bodenschatz, "Finding an H-function distribution for the sum of independent H-function variates," Dissertation, University of Texas, Austin, May 1992.

[27] E. Jones, T. Oliphant, P. Peterson et al., "SciPy: Open source scientific tools for Python," 2001-, [Online; accessed 2016-02-15]. [Online]. Available: http://www.scipy.org/

[28] J. J. More, B. S. Garbow, and K. E. Hillstrom, User Guide for MINPACK-1, 1980.

[29] A. M. Mathai, R. K. Saxena, and H. J. Haubold, The H-function: theory and applications. Springer Science \& Business Media, 2009. 